\title{
Propuestas para el análisis espacial de miniciudades en Centroamérica: más allá del espacio visible
}

\author{
Sabrine Acosta Schnell
}

Artículo

Afiliación: Instituto de Altos Estudios de América Latina (IHEAL), Centre de Recherche et de Documentation des Amériques (CREDA), Unidad de Investigación 7227. Université Sorbonne Nouvelle Paris 3, Paris, Francia.

\section{E-mail: sabrine.acostaschnell@ucr.ac.cr}

Recibido: 4 de septiembre del 2019 Aceptado: 29 de abril del 2020

\begin{abstract}
Sabrine Acosta Schnell
Bachillerato en Geografía en la Universidad de Costa Rica. Máster en Geografía con énfasis en redes, ordenamiento territorial y políticas públicas en la Universidad Federal de Santa Catarina, Brasil. Por concluir el doctorado en Geografía con énfasis en ordenamiento territorial en el Instituto de Altos Estudios de América Latina (IHEAL), Centre de Recherche et de Documentation des Amériques (CREDA), Unidad de Investigación 7227, Université Sorbonne Nouvelle Paris 3, Francia. Docente e investigadora en la Escuela de Geografía, Universidad de Costa Rica. Áreas de interés: mercado inmobiliario, ordenamiento territorial, el proceso de verticalización en Centroamérica, miniciudades y geografía urbana.
\end{abstract}

\begin{abstract}
Resumen
Las miniciudades en Centroamérica son proyectos de uso mixto que combinan espacios residenciales, comerciales y de entretenimiento. Se toman como base dos casos de estudio (Avenida Escazú, en Costa Rica y Ciudad Cayalá, en Guatemala) y se ofrecen propuestas de aproximación teórico-metodológica multidisciplinaria que se podrán utilizar en investigaciones sobre miniciudades. La hibridación de formas urbanas y de funciones contribuye a una complejidad espacial, la cual se sugiere que demanda un análisis que va más allá del clásico concepto dualista de espacialidad. Se abre la discusión a partir de cinco propuestas conceptuales para llevar a cabo el ejercicio de abrir la mente geográfica creativa y tratar sobre nuevos temas y retos urbanos, según las recomendaciones de Soja (2000). Se aborda una selección de autores y métodos, no siempre de la misma línea investigativa, para sugerir las herramientas que permitan analizar las dinámicas espaciales y la convergencia de diversos espacios como el real, el percibido, el concebido, el imaginado, el vivido o el Tercer Espacio de Soja (1996), entre otras propuestas. Se incluye un breve análisis heterotopológico foucaultiano, sin dejar de lado la triple dialéctica de Lefebvre (1974) y otras contribuciones artísticas y literarias. Finalmente, se reflexiona respecto a que el actual proceso de urbanización requiere de nuevos discursos para conceptualizar, en la teoría y en la práctica, las realidades urbanas recientes. El artículo no pretende cerrar la discusión ni concluir de forma restrictiva el análisis espacial, sino que más bien busca provocar la reflexión y la formulación de más preguntas e incógnitas para resaltar la dificultad y necesidad de actualizar y ampliar las herramientas para el análisis de las nuevas dinámicas urbanas.
\end{abstract}

Palabras clave: análisis espacial; heterotopías; miniciudades; Tercer Espacio; uso mixto.

\section{Spatial Analisis Proposals for minicities in Central America: beyond visible space}

\section{Abstract:}

Mini-cities in Central America are mixed-use projects that combine residential, commercial and entertainment spaces. Based on two case studies (Avenida Escazú in Costa Rica and Ciudad Cayalá, in Guatemala), this article offers proposals for a multidisciplinary theoretical-methodological approach that could be used in future research on mini-cities. The hybridization of urban forms and functions contributes to a spatial complexity which is suggested to demand an analysis that goes beyond the classic dualistic concept of spatiality. The discussion opens from five conceptual proposals to carry out the exercise of opening the creative geographical mind to deal with new urban issues and challenges, according to the recommendations of Soja (2000). Addressing a selection of authors and methods, not always from the same research line, this article suggests tools to analyze the spatial dynamics and the convergence of diverse spaces such as the real, the perceived, the conceived, the imagined, the lived or the Third Space from Soja (1996), among other proposals. A brief Foucauldian heterotopological analysis is included, without neglecting Lefebvre's "triple dialectic" (1974) and other artistic and literary contributions. Finally, it is discussed that the current urbanization process requires new discourses to conceptualize, in theory and in practice, recent urban realities. The article does not intend to close the discussion with a restrictive conclusion or with a concluded spatial analysis, but rather aims to provoke consideration and the formulation of more questions and unknowns to highlight the difficulty and need to update and expand the tools for the analysis of the new urban dynamics.

Keywords: spatial analysis; heterotopies; mini cities; Third Space; mixed-use projects. 


\section{Introducción al contexto de Avenida Escazú y Ciudad Cayalá}

$1 \quad$ Este artículo se enmarca en los resultados de una tesis doctoral que, de forma pionera, abre la investigación sobre este tipo de proyectos en la región centroamericana (Acosta, 2017, 2018) Título: Nuevas formas de metropolización en América Central: las miniciudades en San José (Costa Rica) y en ciudad Guatemala (Guatemala). Bajo la dirección de Sébastien Velut. Disponible en: http://www.theses.fr/s156993. Université Sorbonne Nouvelle - Paris III, Institut des Hautes Etudes de l'Amérique latine (IHEAL). Centre de Recherche et de Documentation des Amériques (CREDA) - UMR 7227. Becaria de la Universidad de Costa Rica.
Figura 1. Vista aérea de Avenida Escazú, San José, Costa Rica.

Fuente: Portafolio Inmobiliario (s.f.). as miniciudades en Centroamérica ${ }^{1}$ son proyectos de uso mixto que combinan mínimo tres funciones: la residencial, la lúdica y la comercial. Se propone que estas son una hibridación de lugares y funciones de herencia secular que hoy día convergen y ofrecen una variedad de espacios, actividades y dinámicas. Estos novedosos productos inmobiliarios de iniciativa privada surgen en la región en la última década, con más, 0 menos intensidad, según el país.

Avenida Escazú (figura 1) fue la primera en Costa Rica en adoptar y venderse bajo el concepto de uso mixto, al estilo miniciudad. Su plan maestro incluye una serie de usos y funciones, por ejemplo, el comercio, préstamo de bienes y servicios, centros médicos, residencias, hoteles y restaurantes. Recibe alrededor de 250,000 visitantes al mes, cuenta con aproximadamente 20 restaurantes y bares, 250 habitaciones de hotel, 300 consultorios médicos, más de 100 oficinas de empresas y más de 250 apartamentos tipo lofts (apartamentos en el desván o mezzanine). Asimismo, se planifican 350 nuevas residencias en las siguientes etapas a desarrollar (Lizán, 2018b). Se localiza en el cantón Escazú, en la provincia San José y se trata de una finca de 16 hectáreas que comprende una avenida de un kilómetro de largo (Camacho, 2013).

Por su parte, como ejemplo del Triángulo Norte centroamericano (Guatemala, Honduras y El Salvador), Ciudad Cayalá (figura 2) es la única y más grande miniciudad hasta ahora construida en Guatemala, en Zona 16. El plan maestro tiene una amplia oferta residencial que incluye viviendas en condominios, lotes, apartamentos y lofts, donde habitan alrededor de 1,300 familias (Morales, 2018). Además, se ofrecen viviendas en formato courtyard (patio interno), en edificios de no más de seis pisos de altura y brinda más de 150 ofertas de tiendas, cafés y restaurantes en su directorio comercial.

Ambas miniciudades se seleccionaron para ejemplificar proyectos de uso mixto que incluyen el componente residencial y que son, al mismo tiempo, de diversos tamaños, planificadas en diversas etapas a lo largo de las décadas. Estos proyectos se localizan en ciudades capitales, las cuales participan activamente de los procesos de globalización y han presentado mercados inmobiliarios altamente activos y competitivos, con la promoción e introducción de cambios en las ofertas urbanas.

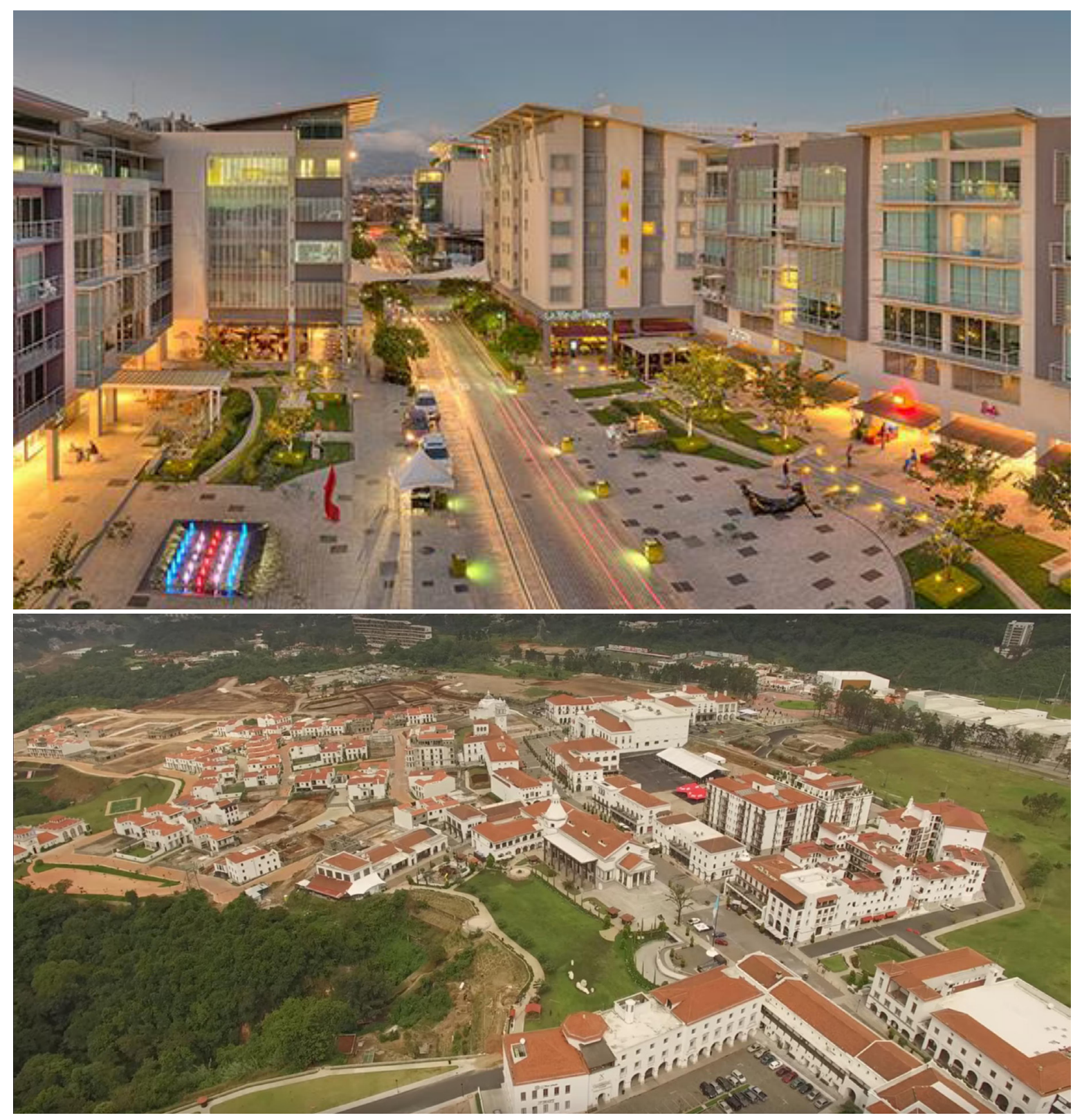


A partir de estos dos casos de estudio, se plantean cinco abordajes conceptuales a modo de herramientas para analizar la complejidad espacial que se asocia a las miniciudades como formas urbanas que son resultado de cambios en la estructura socioeconómica de las ciudades actuales (Acosta, 2018). Las ideas planteadas no pretenden ser exhaustivas, sino detonantes para ampliar las discusiones y debates sobre la complejidad urbana.

Históricamente, los nexos entre las tipologías polifuncionales de entretenimiento han influido en la morfología de la ciudad, en las relaciones de esta con los urbanitas y entre estos. Los paisajes urbanos y comerciales también cambian, en respuesta a las diferentes formaciones sociales, configuraciones económicas y políticas (Derycke, Huiriot y Pumain, 1996). El ocio y el entretenimiento han sido siempre un factor significativo en el desarrollo económico, social y territorial.

Asimismo, han existido complejas relaciones entre el paisaje, el gusto, las clases sociales y el poder, desde el siglo XVII hasta los espacios hoy (Schenker, 2002; Cosgrove, 1998). Además, Clavé (2007), Young (2002), Hardwood (2002), De Moncan (2012), Benjamin (1939), Cosgrove (1998) y Schneker (2002) han investigado detalles históricos para fundamentar las tipologías desde la perspectiva funcional y simbólica e identificar nuevas articulaciones sociales en los espacios de ocio. Actualmente, el entretenimiento sigue teniendo mucha importancia en la construcción social del espacio, pues posee una función integradora de espacios comerciales desde mucho antes que Víctor Gruen, "inventor" del mall, publicara su obra Shopping towns USA, The planning of shopping centers (1960) e integrara el factor entretenimiento en sus nuevas propuestas de centros comerciales en los suburbios estadounidenses.

El ocio yel entretenimiento son significativos catalizadores del desarrollo enlaseconomías actuales (Clavé, 2007) y juegan un papel importante básico en las miniciudades, en las que, adrede, los límites entre entretenimiento y consumo desaparecen. La polifuncionalidad de estas y el retailtainment (venta de entretenimiento, emociones y sensaciones) han convertido los espacios comerciales en "verdaderos centros sociales", según Cristian Muñoz, gerente retail corporativo del Mall Plaza, operador de malls en Chile, Colombia y Perú (Groover, 2015).

Otro factor a incluir en la recapitulación histórica son las transformaciones en la percepción sobre el tiempo libre para entretenerse, consecuencia de la organización social del trabajo después de la Revolución Industrial (Clavé, 2007). Esta idea es esencial en la comprensión de la emergencia de lugares de entretenimiento hibridados con otras funciones. En otras palabras, los paisajes, los gustos y las necesidades cambiaron a lo largo de los siglos, y se propone analizar las miniciudades como una propuesta inmobiliaria que se adapta a las relaciones urbanas, a modo de reacción frente a un momento particular en la historia. Así pues, las miniciudades son productos inmobiliarios vendidos y mercadeados en una respuesta a las configuraciones de los sistemas urbanos actuales.

Las metrópolis en Centroamérica albergan una reducida cantidad y calidad de espacios compartidos, unas más que otras, y las herramientas propuestas pretenden contribuir a verificar y re-entender el centro comercial y las miniciudades como polos multifuncionales en las cuales el entretenimiento sirve para crear nuevos lugares, nuevas relaciones sociales y nuevas dinámicas de consumo. Este artículo no pretende cerrar la discusión ni concluir de forma exhaustiva, sino más bien abrir más las posibles herramientas de análisis y ampliar los cuestionamientos sobre el tema, para enfatizar la complejidad urbana actual.

Se sugiere la necesidad de repensar, mantener creativa y actualizada la imaginación geográfica crítica, abierta a posibilidades de redefinición y expansión de los conceptos más allá de la visión tradicional dualista, según Soja (1996). En este contexto, se proponen, de forma sintética, las siguientes cinco herramientas conceptuales para las investigaciones futuras sobre miniciudades:

La trialéctica de Lefebvre (1974) y el thirding-as-Othering de Soja (1996).

Un examen heterotopológico foucaultiano de los espacios, con un análisis de los espejos y las vitrinas como ventanas a otros mundos. 
La hiperrealidad de Baudrillard (2016).

El plan maestro como la anticipación que le da vida al territorio.

Un análisis literario de obras, el cual facilita la comprensión de mundos de inmersión paralelos, según se sugiere que acontece en las miniciudades en Centroamérica.

Cada sección del artículo corresponde a una propuesta que, se reitera, no pretende llegar a una conclusión puntual, sino sumar a las posibilidades de estudiar el espacio. No se llega a realizar el análisis espacial, pero se trata de evaluar las ideas planteadas para repensarlas como posibles herramientas para futuros estudios. Se inicia con la teoría sobre "el otro espacio", pasando por las heterotopías, a modo de herramienta que permite la comparación entre dos espacios y, finalmente, se propone el abordaje de la literatura donde el lector podrá visualizar y aplicar los conceptos e ideas previamente discutidos.

\section{Un espacio más: I'Autre / the Other / el Otro}

Al entrar a la tercera década del siglo XXI, se hacen evidentes las complejidades de lo social, lo histórico y lo espacial. La magnitud del reto para ir más allá del dualismo hizo que Soja (1996) propusiera el concepto de Tercer Espacio como un término flexible y un intento de capturar esa constante dinámica en ideas, eventos, apariencias y significados. Es una complejidad entretejida que se puede identificar en los espacios híbridos de las miniciudades.

Según el planteamiento del citado autor, el Tercer Espacio es una invitación para que la imaginación geográfica pueda expandirse y envolver la multiplicidad de perspectivas que los preconceptos epistemológicos tradicionales han considerado incompatibles. Es una forma de pensar el espacio e introducir más variables al típico dualismo que ha caracterizado los imaginarios espaciales. Esa variable extra es propuesta por Soja (1996) como thirding-as-othering. Ahora, la percepción binaria se somete a un proceso de reestructuración, donde se recombinan y se extienden las visiones espaciales.

También se puede traer a la discusión la propuesta de Lefebvre (1974) acerca de los tres espacios: el primero ( "el percibido"), el segundo ("el concebido") y el tercero ("el vivido"). Esta propuesta, para entender la teoría y la práctica, es esencial al analizar la dialéctica de lo social-histórico. Desde la tercera perspectiva, se introduce la variable espacial para lo que vendría a ser la propuesta de Soja (1996) de la "triple dialéctica" o la "trialéctica", que incluiría la historicidad, la socialidad y la espacialidad. En este momento, se propone el concepto de dialectique de triplicité (Lefebvre, 1974) en el contexto de la producción del espacio o las relaciones entre el espacio, el tiempo y el ser social.

Lefebvre (1974) critica toda dicotomía o formas de lógica binaria. En un contexto donde las oposiciones no son suficientes, él propuso que /l y a toujours I'Autre, siempre está el Otro. Para romper la lógica del "este o aquel", él optó por el "y". De esta forma, se tiene I'Autre/the Otherlel Otro en lo que vendría a proponer como la "trialéctica del espacio". Con esta metodología, se pueden identificar "otros" espacios en las miniciudades que interactúan con la metrópolis.

Así pues, para Lefebvre (1974) el "espacio vivido" es la simple combinación entre el "espacio real" y el "espacio imaginado". A partir de esa visualización, Soja (1996) propuso que el Primer espacio es todo aquello concreto y material, aquello que podría ser empíricamente medido y mapeado y el Segundo espacio incluye las ideas y las formas mentales y cognitivas de concebirlo. De esta manera, tales concepciones coinciden con el espacio "real" e "imaginado" de Lefebvre (1974).

Esta metodología de la "trialéctica" propuesta por Soja (1996) pretende enfatizar más la parte espacial del razonamiento clásico dialéctico de lo histórico-social. A su vez, de forma exploratoria, pretende inspirar el análisis de las miniciudades para observarlas como una nueva forma de redefinir la espacialidad de la vida del ser humano y de ir más allá de los binarismos analíticos. ¿Sería de utilidad esta propuesta conceptual para considerar los posibles usos, actores, localizaciones y dinámicas entre las miniciudades 
y la metrópolis? Sí, se considera un ejercicio que podría replantear la multiplicidad de visiones sobre los emergentes espacios urbanismo contemporáneo. Seguidamente, se analizan las heterotopías como herramienta para abordar una perspectiva más dualista.

\section{Heterotopías y las miniciudades: una herramienta para reconsiderar las dinámicas en la ciudad}

Otro concepto que podría inspirar el análisis de las miniciudades parte de las propuestas de Foucault (1984) (Acosta, 2018). Este autor aseveró que el ser humano no vive en un espacio vacío, sino en uno definido por una serie de relaciones entre los sitios y los que considera heterotopías. En su obra Des espaces autres (1984) explica que los espacios pueden ser utopías (emplazamientos sin lugar real) o heterotopías (opuestas a las utopías, como lugares reales localizables). Una de las características de las heterotopías resulta ser el poder que tienen para yuxtaponer diversos lugares que podrían considerarse incompatibles en sí mismos.

Al aplicar esa herramienta de estudio a las ciudades en Centroamérica, se podrían considerar estas tipologías urbanas como heterotopías que representan esa totalidad de la metrópolis (Acosta, 2018) desde una perspectiva binaria o comparativa. Este concepto permitiría analizarlas como un mundo dentro de otro, donde se puede encontrar un oasis de paz, seguridad y limpieza en medio del conglomerado urbano. Son una realidad paralela que invita a divertirse, vivir, comer y trabajar en un solo lugar; pretenden ofrecer todo lo necesario para desconectarse del exterior (Acosta, 2018).

Así pues, las miniciudades poseen, a la vez, submundos con su propia realidad, en los que se puede elegir para sumergirse: hacia las vitrinas, los espacios "públicos" (o compartidos), o las residencias. Las heterotopías pueden brindar la visión de estos proyectos como mundos paralelos y envolventes para los usuarios, habitantes, visitantes y soñadores, que tienden a "internalizar" las ventajas y "externalizar" los inconvenientes, para utilizar las palabras de Monnet (2011, p. 6), en el contexto de la discusión de la "ville de demain" (la ciudad de mañana). Es una forma de abrir la investigación a los diversos discursos y actores con sus correspondientes papeles y objetivos. Se analizan, a continuación, las vitrinas, superficies que reflectan el mundo idealizado para los urbanitas, de parte de los promotores.

\section{Entrando en los espejos y vitrinas: un mundo que sí existe.}

El análisis de los espejos, realizado por Foucault en las obras de arte, también es una valiosa herramienta que se propone para el análisis espacial de los usos mixtos en las miniciudades vistas como heterotopías. En esta sección se ejemplifica la complejidad de la simultaneidad espacial en los espejos representados en las pinturas artísticas, comparadas con el papel en las vitrinas de las miniciudades.

¿Cómo podría contribuir este debate al análisis de las miniciudades? En cuanto al análisis de las miniciudades, este debate, al retomar la visión dualista que proponen las heterotopías, podría permitir una comparación de usos y necesidades (reales o vendidas) en la población. Por ejemplo, según Foucault, el espejo resulta una utopía y una heterotopía al mismo tiempo. Por un lado, es un lugar que no existe; es un espacio irreal que solo existe detrás de una superficie. El "yo", se puede mirar donde en realidad no está. Por el contrario, el espejo también es real. Existe y convierte este lugar en real y relacionado con todo lo que lo rodea, como una heterotopía. Esta discusión permite mostrar la existencia de una diversidad espacial, de combinaciones y complejidades en las miniciudades en Centroamérica, en las que los usuarios, transeúntes, residentes, trabajadores o turistas pueden combinarlas según sus deseos, necesidades y roles en la dinámica urbana. Esto muestra que se requieren nuevos abordajes de estudio y conceptos actualizados desde la perspectiva de lo real, lo imaginado, lo vivido, lo pensado, lo posible, lo deseado. 
Si se propone el arte como otra herramienta de análisis de los espejos y mundos idealizados, la pintura de Jan van Eyck, el Retrato de Arnolfini (figura 3), está cargada de realismo y simbolismo con el icónico espejo convexo que parece mostrar otro mundo paralelo o reflejado desde otra perspectiva del que se pinta en primera plana en el mundo real. Se trata del mismo espacio, pero uno es real y el otro es simplemente una imagen. En la pintura holandesa era común encontrar espejos que duplicaban y reduplicaban lo que aparecía en el cuadro, pero a modo de un espacio modificado, curvado por su superficie. Resultaba un espacio irreal basado en el reflejo de una realidad. Igual que en el caso de las miniciudades, estas pueden ser vistas como un reflejo idealizado de una ciudad utópica que no existe; sin embargo, es posible entrar, residir, entretener y usar tanto como en la ciudad real.

Criticadas por muchos por ser una simple imagen falsa de un espacio urbano fabricado, las miniciudades existen al igual que las imágenes reflejadas por el espejo de Jan van Eyck (figura 3) o, por qué no, en el de Las Meninas de Diego Velásquez (figura 4).
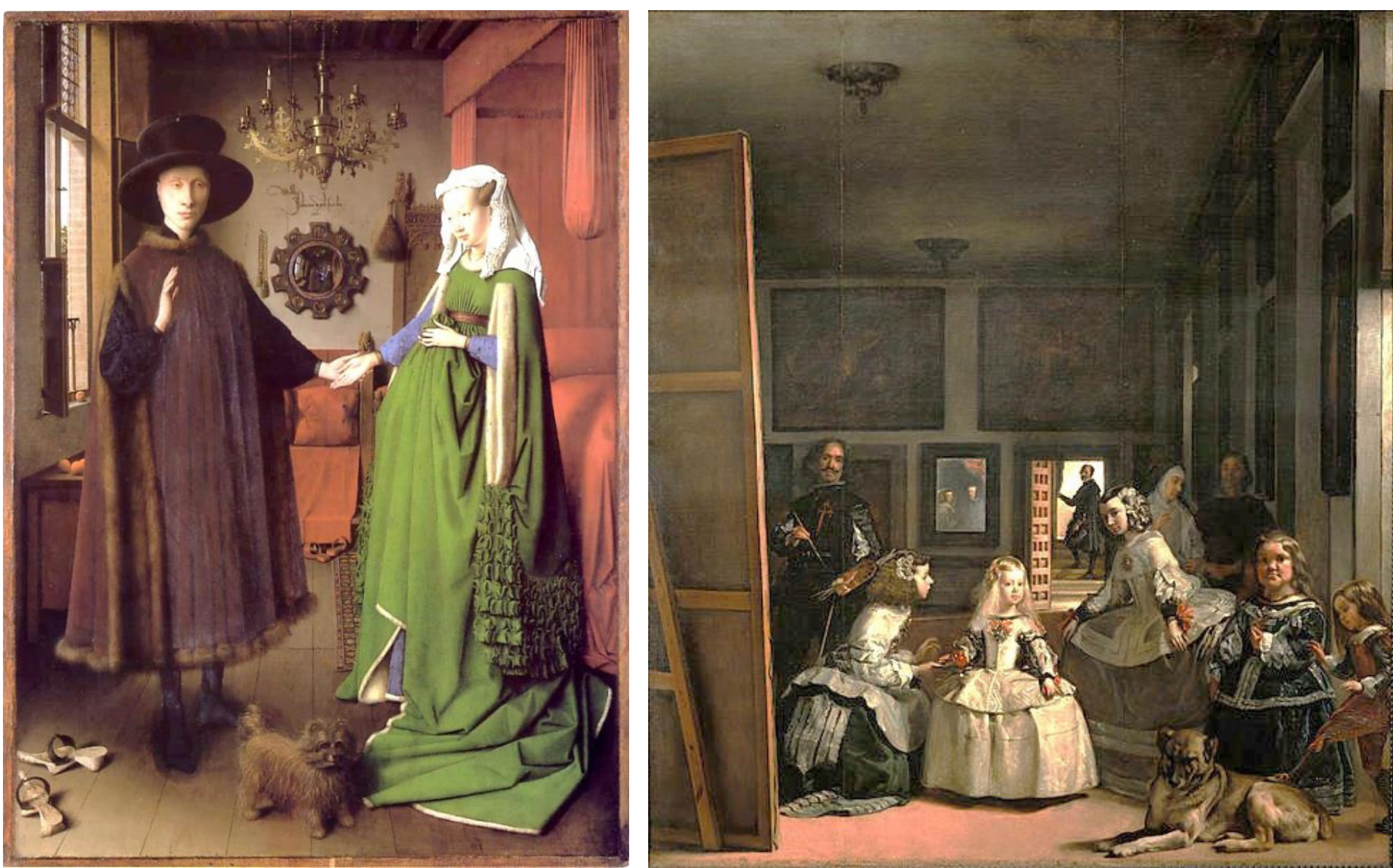

Figura 3. Arnolfini Portrait (1434, van Eyck). Fuente: National Gallery (2019).

Figura 4. Las Meninas (1625, Velásquez) Fuente: Museo Del Prado (2019).
Esta segunda obra es analizada por Foucault (2007) en su primer capítulo del libro Las palabras y las cosas. En concordancia con el autor, al igual que en las miniciudades, cabe la pregunta: “¿vemos o nos ven?". Los contemplados o los contempladores son atrapados por el pintor, quien está representado en el mismo cuadro. Son obligados a entrar en el cuadro para ser retratados sobre la superficie de la tela o del lienzo. De la misma forma que los personajes de Las meninas, los usuarios, visitantes y residentes de las miniciudades son invitados y atrapados para entrar en ese mundo, en ese espacio de inmersión que seduce a permanecer ahí para siempre.

Al igual que la mirada del autorretrato de Velásquez, las miniciudades aceptan aquellos modelos o espectadores que surjan o visiten. Según Foucault (2007), el contemplador y el contemplado se intercambian. Al caminar por Ciudad Cayalá o Avenida Escazú, es posible que la persona sea contemplada por contempladores, visitantes o residentes. Todos son contempladores al tiempo que son contemplados. Puede ser a partir de una dimensión real o percibida, o desde una dimensión idealizada (para unos sin suficiente poder adquisitivo), desde una dimensión soñada (las vitrinas) o desde otras dimensiones que se podrían incluir en la clásica tríada de lo percibido, concebido y vivido. Estos diversos espacios cambian según el rol, ya sea visitante, residente, trabajadores 0 compradores. Desde cada una de esas perspectivas, el contemplado o el contemplador pueden tener una amplia gama de combinaciones de espacios. Los que para unos son 
espacios vividos o percibidos, para otros pueden ser apenas concebidos (Lefebvre, 1974), incluso pensados (Bozzano, 2004) o, simplemente, deseados (Acosta, 2018).

Esta analogía del espejo con los diversos mundos reflejados se puede relacionar con las vitrinas de las tiendas en las miniciudades. Estas reflejan un mundo que no es real, pero los consumidores se ven ahí inmersos. Ahora bien, las personas sueñan con escapar a otro lugar y, aunque no puedan acceder a este, sino solo contemplarlo, lo hacen estando ya inmersos en una dimensión que es la miniciudad. Este tipo de reflexión detona las investigaciones sobre el motivo de crear miniciudades, quién las crea, para qué y para quiénes.

Al igual que con el ejemplo de los espejos representados en las pinturas artísticas, se pueden analizar las superficies reflectantes en las miniciudades, a partir de preguntarse quién está siendo reflejado si la persona misma o una idealización de esta y quiénes están a su alrededor. No es posible verlos directamente pero sí a través de un reflejo. Se ve el mundo real reflejado en un vidrio y sobrepuesto al mundo idealizado de lo expuesto en la vitrina. A partir de estas herramientas se podría cuestionar si las miniciudades son propuestas del mercado inmobiliario vendidas a manera de "solución" a los retos actuales metropolitanos.

Según, comenta Foucault (2007) sobre el espejo de Velásquez, este permite un juego entre el exterior y el interior. Es una forma de yuxtaponer un contenido ilusorio y verdadero, de la misma forma que acontece en la dinámica entre las miniciudades y la metrópoli. Se podrían analizar las miniciudades como opuestos o como espacios deseados versus la metrópolis real. Al igual que en Las Meninas, en las miniciudades hay varios espectadores y observados, actores, momentos y perspectivas en un mismo espectáculo.

En la obra está el pintor, los modelos y el visitante en la puerta. Todos se asoman a la escena y participan del espectáculo desde su perspectiva. Asimismo, se pueden agregar los personajes reflejados en el espejo, el cual parece multiplicar y refractar la realidad. Existe también la realidad capturada en la tela, la cual, de forma semejante al espejo, parece perpetuar la realidad, solo que de forma definitiva. El espejo (al igual que en las vitrinas) permite brindar un mundo inmediatamente paralelo a la realidad, que ayuda a cuestionarse, en el caso de las miniciudades: ¿es un mundo mejor 0 peor?, ¿más limpio, más seguro, deseado o excluyente? En la pintura, además, parece que no todos los personajes pueden "entrar" en el espejo, al igual que las personas no pueden entrar en las vitrinas de las miniciudades, pero pueden ver y soñar con espacios reflejados, refractados, duplicados o modificados del real.

A partir de lo anterior, cabe cuestionarse cuáles son los límites entre la mente y la realidad. Seguidamente, este punto se aborda desde el punto de vista de los trucos visuales, para generar más preguntas que contribuyan al debate.

\section{Un truco visual e "hiperreal"}

La convergencia o divergencia de la realidad y la fantasía en las miniciudades también se puede abordar desde la perspectiva de hiperrealidad, propuesta por Jean Baudrillard (2016) (Acosta, 2018). Las miniciudades son como un mundo donde no se distinguen los límites entre la realidad y el simulacro. Esto detona discusiones sobre el afán por estar constantemente reproduciendo lo real y lo que la sociedad ha perdido: los valores, los momentos de éxito político y económico. Por tal razón, algunas miniciudades (por ejemplo, Ciudad Cayalá) añoran el pasado idílico (Acosta, 2018) a través de la tematización en sus diseños y arquitecturas (Lukas, 2007, 2008, 2013; Gottdiener, 2007).

Se crea una hiperrealidad y "cuando lo real ya no es lo que era, la nostalgia cobra todo su sentido" (Baudrillard 2016, p. 19); ya sea el pasado colonial, el pasado griego y romano o también el éxito de las ciudades mayas. Es una forma de recrear el pasado y perder noción de lo que es real. Se asemeja a sumergirse en las lecturas de las Leyendas de Guatemala de Miguel Ángel Asturias (1930) para dejarse llevar por el mundo mágico del pasado. El usuario de las miniciudades elimina los nexos temporales 
al observar la mezcla de milenarios estilos arquitectónicos. Entra en un universo para viajar hacia el pasado venerado y se identifican capas temporales de ciudades antiguas que se amalgaman y brotan en la contemporaneidad. En el caso de las miniciudades costarricenses, estas más bien optan por una tematización que sigue una línea más futurista, de arquitecturas sencillas que aluden al progreso, a lo fashion cosmopolita.

Las miniciudades son un espacio donde adrede se confunde lo real con lo simulado (Acosta, 2018). Según Baudrillard (2016), el hiperrealismo de la simulación es una metáfora de la realidad, la cual tiene "un alucinante parecido de lo real consigo mismo" (p. 53). En palabras de este autor, es un

[...] corto circuito fantástico: lo real es hiperrealizado. Ni realizado, ni idealizado: hiperrealizado. Lo hiperreal es la abolición de lo real no por destrucción violenta, sino por asunción, elevación a la potencia del modelo. [...] El modelo opera como esfera de absorción de lo real. (p. 189)

Precisamente, este modelo hiperrealizado son las miniciudades. Se cuestiona hasta dónde Avenida Escazú y Ciudad Cayalá son planificadas como un microcosmos que alberga lo necesario para vivir, entretenerse, trabajar, comer y comprar. Según el discurso de sus promotores, no hay necesidad de conectarse con el mundo externo. Ese es su mundo, pero ya no se sabe cuál es el mundo real. Es un juego entre la realidad y la hiperrealidad.

Ahora bien, ¿cuál es el discurso de los diferentes sectores de la población? Según el lenguaje publicitario, el mundo externo se replica para no tener necesidad de interactuar con él. Se replica higienizando su realidad urbana: planifica y elimina los altos índices de violencia, la acechante contaminación, la ausencia de espacios de recreo, las funciones y actividades urbanas desconectadas, entre otras amenazas. Lo que Monnet (2011) llamó externalizar inconvenientes, en alusión a la "paradoja del laberinto".

Así mismo, cabe preguntarse si toda la población está incluida en esta "solución", pues este tipo de cuestionamientos son los que se detonan a partir de la función de crear hiperrealidades. Sin embargo, muchos no logran entender la razón de estos espacios hiperrealizados. Las miniciudades son mundos de inmersión planificados adrede, no son un falso intento de simulación. Se debe ver más allá y distinguir los discursos. No se trata de un intento fallido de "Disneyficar" (Sorkin, 1992), ni un espacio solo para que se vea "bonito", o "semejante" a la ciudad "afuera", sino de intercambiar las realidades (Acosta, 2018). Ocultar una realidad por otra que ya no existe dentro de sus paredes. La realidad y la hiperrealidad cambian de papeles. Ahora, Ciudad Cayalá y Avenida Escazú son la realidad que "debería de ser". Según Baudrillard (2016), es intentar salvar el principio de realidad. Son un mecanismo de "disuasión" para "[...] regenerar a contrapelo la ficción de lo real" (p. 31).

Estos proyectos urbanos son un "truco visual" planificado adrede para "producir un simulacro, con plena conciencia del juego y del artificio" (Baudrillard, 2016, p. 4). Los argumentos críticos desinformados que aluden a productos heredados del proceso de globalización llevan a una incomprensión intelectual de las formas urbanas (Salcedo, 2003; Salcedo y de Simone, 2013), impiden ver más allá de la supuesta "falsedad de su carácter urbano" (Acosta, 2018). No se pretende replicar exactamente el mundo exterior, sino "tamizarlo", pues no tendría sentido realizar una copia. Este simulacro es consciente con diversos grados de autenticidad y busca filtrar e imitar solo aquellos aspectos que los desarrolladores y diseñadores escogieron como atrayentes para los usuarios y residentes en su equis realidad urbana (Acosta, 2018). Así, esta propuesta conceptual permite levantar cuestionamientos sobre discursos, actores, motivos y técnicas para lograrlo. En la siguiente sección se analiza, a partir del plan maestro, cómo este se anticipa a la creación de espacios, momentos, usos, funciones y dinámicas, y se utiliza como ejemplo un extracto literario.

\section{"Precesión de los simulacros"}

Se propone el análisis de Baudrillard (2016), quien comenta que la simulación se genera a partir de algo real sin origen. El espacio imaginado es el que precede al espacio real. En el caso de las miniciudades, el mapa (o el plan maestro) es el que 
le da vida al territorio. El mapa "precede" al territorio y no al contrario. ¿Ocurriría esto con la inversión del sector público también? En los proyectos privados primero existe el mapa y luego las miniciudades. Es lo que Baudrillard (2016) llama "precesión de los simulacros".

Al orientar el análisis espacial a partir de la literatura, se retoma el tema de la simulación en el cuento corto "Del rigor en la ciencia" de Borges (1999), donde el "arte de la cartografía" creó un mapa para incluir toda la ciudad, lo cual hace recordar los planes maestros de las miniciudades. La brevedad de este cuento corto permite citarlo a continuación:

En aquel Imperio, el Arte de la Cartografía logró tal Perfección que el Mapa de una sola Provincia ocupaba toda una Ciudad, y el Mapa del Imperio, toda una Provincia. Con el tiempo, esos Mapas Desmesurados no satisficieron y los Colegios de Cartógrafos levantaron un Mapa del Imperio, que tenía el Tamaño del Imperio y coincidía puntualmente con él. Menos Adictas al Estudio de la Cartografía, las Generaciones Siguientes entendieron que ese dilatado Mapa era Inútil y no sin Impiedad lo entregaron a las Inclemencias del Sol y los Inviernos. En los Desiertos del Oeste perduran despedazadas Ruinas del Mapa, habitadas por Animales y por Mendigos; en todo el País no hay otra reliquia de las Disciplinas Geográficas. SUÁREZ MIRANDA: Viajes de varones prudentes, Libro cuarto, Cap. XLV, Lérida, 1658.(Borges, 1999, p. 119)

En el cuento se destaca el poder del Imperio y el encanto de los simulacros, al crear, recrear y replicar una realidad. Estos vestigios del mapa quizás sean los de un mapa colonial, de una ciudad inicial, la ciudad que se recuerda con melancolía, de la supuesta ciudad perfecta; ese San José colonial, esa Guatemala colonial. La idea de ciudad perfecta es la que se trata de re-crear en otro mapa, como lo hicieron los "Colegios de Cartógrafos" de Borges pero, en este caso, el mapa (el plan maestro de las miniciudades) precede al territorio (figura 5).

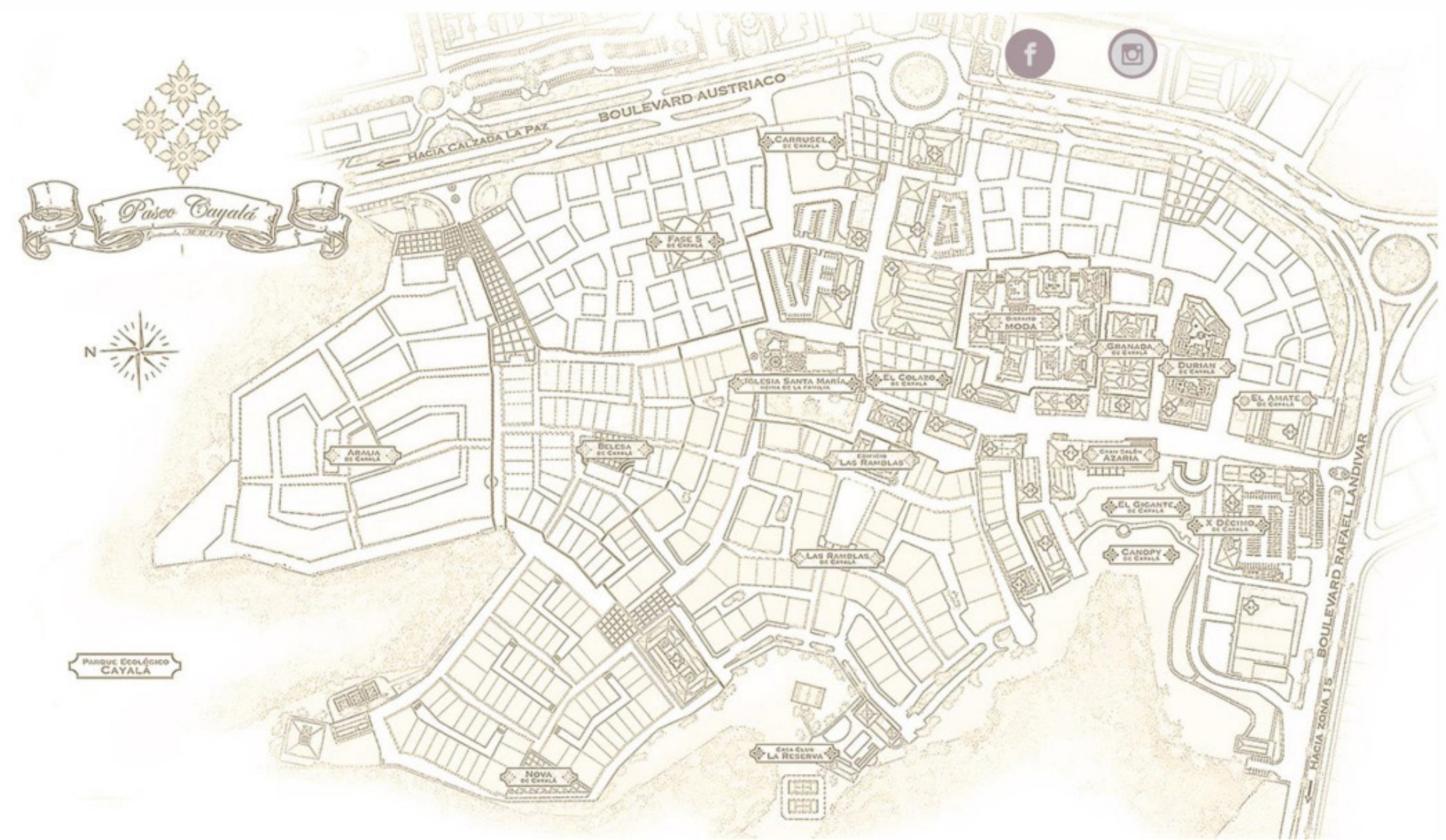

Figura 5. Mapa de Ciudad Cayalá, Guatemala. Fuente: Cayalá Residencial. (s.f.).
Este extracto literario permite formular más preguntas sobre la planificación de miniciudades, sus públicos meta, su relación (real o ficticia) con la metrópolis, los discursos publicitarios para vender el orden, lo planificado, lo seguro, lo limpio y lo agradable. Así pues, se plantea la interrogante acerca de cómo se vende el espacio 0 ciudad perfecta. 


\section{El mundo ilusorio de Don Quijote}

Se ha sugerido, desde varias disciplinas, que las miniciudades en Centroamérica son un conjunto de espacios donde la línea entre la realidad y la ilusión resulta borrosa. Incluso, al igual que en las heterotopías, no se sabe si la realidad está entre intramuros 0 extramuros. Se le convence al urbanita, quien es usuario o residente, que la realidad, o lo que "debería ser", está dentro de la miniciudad. Los promotores y el marketing se encargan de hacer aceptar o creer esa nueva gama de espacios tan reales como concebidos, tan reales como las aventuras ilusorias de Don Quijote.

Al igual que el hidalgo y Sancho Panza cabalgando por algún lugar de La Mancha, así son las caminatas, aventuras y vidas con las calles y plazas en las miniciudades, llenas de espacios imaginados, pensados, soñados e hiperreales. Más allá de solo crear un espacio tematizado o ilusorio, tanto los desarrolladores inmobiliarios como Miguel de Cervantes, tratan de hacer ver de qué forma, a través de la ilusión, se pretende cambiar a una realidad mejor. Cabe cuestionarse si las miniciudades son intentos de proyectos para tratar de promover una mejor ciudad, pues estas reflexiones surgen a partir de la lectura literaria, que abre la mente geográfica e invita al usuario, al lector y al investigador a estar abiertos a nuevas sugerencias y necesidades de estudio.

La escena de los molinos ejemplifica cómo Don Quijote, con su locura, intentaba cambiar la realidad. Las miniciudades tematizadas, planificadas e higienizadas venden una realidad que, supuestamente, se termina viendo más atractiva, limpia, segura e idílica. Dice la escena:

En esto, descubrieron treinta o cuarenta molinos de viento que hay en aquel campo; $y$, así como don Quijote los vio, dijo a su escudero:

- La ventura va guiando nuestras cosas mejor de lo que acertáramos a desear, porque ves allí, amigo Sancho Panza, donde se descubren treinta, o pocos más, desaforados gigantes, con quien pienso hacer batalla y quitarles a todos las vidas, con cuyos despojos comenzaremos a enriquecer; que ésta es buena guerra, y es gran servicio de Dios quitar tan mala simiente de sobre la faz de la tierra.

- ¿Qué gigantes? -dijo Sancho Panza.

-Aquellos que allí ves -respondió su amo- de los brazos largos, que los suelen tener algunos de casi dos leguas.

-Mire vuestra merced -respondió Sancho- que aquellos que allí se parecen no son gigantes, sino molinos de viento, y lo que en ellos parecen brazos son las aspas, que, volteadas del viento, hacen andar la piedra del molino.

-Bien parece -respondió don Quijote- que no estás cursado en esto de las aventuras: ellos son gigantes; $y$ si tienes miedo, quítate de ahí, y ponte en oración en el espacio que yo voy a entrar con ellos en fiera y desigual batalla. (Cervantes, s.f., p. 75)

Este análisis pretende mostrar de qué modo, a través de la literatura, se puede ver el intento de un autor por problematizar los diversos espacios que rodean a las personas. Parece que la ficción o la ilusión se tornan realidad cuando se recurre a la imaginación y a los proyectos como las miniciudades para "transportar" del mundo real o percibido hacia el mundo ideal, concebido, imaginado o pensado que tanto se anhela y se "necesita".

En las miniciudades se trata de ver los diferentes espacios a través de una ventana o a través de un muro. Se puede ver un espacio ilusorio al otro lado de la "entrada" 0 afuera de las ventanas; ver desde afuera o desde adentro de la misma miniciudad. La figura 6 ejemplifica cómo se puede apreciar la ilusión desde otro espacio, insinuando que se está mirando desde otro molino que también hace parte de la misma ilusión. Según planteaban las heterotopías de Foucault (1984), cabe preguntarse dónde están los límites de la ilusión y lo real y si se está adentro o se está afuera.

Las técnicas de tematización, marketing, arquitectura y diseño en general, son recursos para construir esa realidad dentro de otra. Así pues, ¿una pequeña ciudad o una miniciudad dentro de una metrópolis podría plantearse como la estrategia de llegar a visualizar el mundo o ciudad ideal?

Estos cuestionamientos podrían orientar hasta dónde es posible que muchos usuarios 
Figura 6. Perspectiva de los molinos de viento de Consuegra, Toledo.

Fuente: Acervo de la autora (2016).

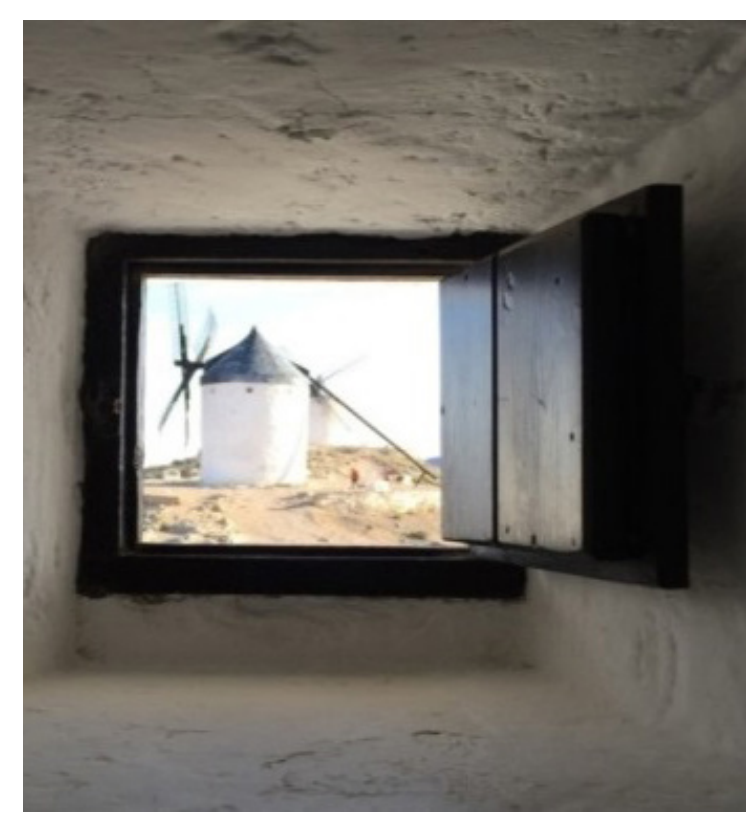

y visitantes, aunque no pertenezcan al segmento social con el poder adquisitivo para consumir o vivir en estas miniciudades de alto perfil, pueden hacer como Alonso Quijano, crear su personaje y vivir el espacio a partir de Don Quijote. Es una forma de escapar de la realidad. Se mezclan tiempos y espacios en una ficción idealizada donde, Don Quijote y los usuarios/residentes están capturados entre dos mundos: entre ciudad Guatemala y Ciudad Cayalá; entre ciudad San José y Avenida Escazú.

\section{Miniciudades: el Aleph centroa-}

Un último ejemplo de herramienta para el análisis espacial es el realismo mágico de Borges (1983), autor que lleva al lector a conocer "[...] el lugar donde están, sin confundirse, todos los lugares del orbe" (p. 166); se trata de El Aleph. En su cuento, titulado con el mismo nombre, el protagonista descubre un lugar en el sótano de la casa de su amigo donde se pueden ver todos los lugares de la Tierra:

En la parte inferior del escalón, hacia la derecha, vi una pequeña esfera tornasolada, de casi intolerable fulgor. Al principio la creí giratoria; luego comprendí que ese movimiento era una ilusión producida por los vertiginosos espectáculos que encerraba. El diámetro del Aleph sería de dos o tres centímetros, pero el espacio cósmico estaba ahí, sin disminución de tamaño. Cada cosa (la luna del espejo, digamos) era infinitas cosas, porque yo claramente la veía desde todos los puntos del universo. Vi el populoso mar, vi el alba y la tarde, vi las muchedumbres de América, vi una plateada telaraña en el centro de una negra pirámide, vi un laberinto roto (era Londres), vi interminables ojos escrutándose en mí como en un espejo, vi todos los espejos del planeta y ninguno me reflejó, [...] vi racimos de nieve, tabaco, vetas de metal, vapor de agua, vi convexos desiertos ecuatoriales y cada uno de sus granos de arena, [...] vi la noche y el día contemporáneo, vi un poniente en Querétaro que parecía reflejar el color de una rosa en Bengala, vi mi dormitorio sin nadie, vi en un gabinete de Alkmaar un globo terráqueo entre dos espejos que lo multiplicaban sin fin, vi caballos de crin arremolinada, en una playa del Mar Caspio en el alba, vi la delicada osatura de una mano, vi a los sobrevivientes de una batalla, enviando tarjetas postales, vi en un escaparate de Mirzapur una baraja española, vi las sombras oblicuas de unos helechos en el suelo de un invernáculo, vi tigres, émbolos, bisontes, marejadas y ejércitos, vi todas las hormigas que hay en la tierra [sic] vi un astrolabio persa, [...] vi el Aleph desde todos los puntos, vi en el Aleph la tierra [sic], vi mi cara y mis vísceras, vi tu cara, y sentí vértigo y lloré, porque mis ojos habían visto ese objeto secreto y conjetural, cuyo nombre usurpan los hombres, pero que ningún hombre ha mirado: el inconcebible universo. (Borges, 1949)

El Aleph de Borges invita a explorar la alegoría de las infinitas complejidades del espacio y del tiempo (Soja, 1996). Agrega otra visión al espectro de conceptualizaciones sobre el espacio que se ha venido explorando en este artículo para proponer conceptos e ideas que orienten la investigación de las miniciudades. Al igual que El Aleph, estas tienen todo en un mismo lugar/proyecto. Se parecen al microcosmos de los jardines persas o japoneses. Hay espacios para ocio, para trabajar, para comprar, para vivir, para caminar, para perderse y para descubrir. Las miniciudades se venden como un producto inmobiliario que se asemeja a un universo, un espacio ideal infinito donde se puedan satisfacer todas las necesidades, básicas y de abundancia y exuberancia.

El reto de entender la citada obra de Borges en relación con las miniciudades recuerda el Third Space de Soja (1996), discutido a inicios de este artículo. Es un mundo donde convergen todos los espacios, es un objeto secreto lleno de ilusiones y alusiones, un lugar común para "todos", aunque no puede ser completamente observado ni analizado. Según Soja (1996), el Third Space es donde se encuentra la subjetividad y la objetividad, lo abstracto y lo concreto, lo real y lo imaginado, lo conocido y lo inimaginable, lo repetitivo 
y lo diferencial, mente y cuerpo, lo consciente y lo inconsciente, la vida cotidiana y la interminable historia, entre otras ideas. Se pueden obtener diversas preguntas sobre inclusión y exclusión; por ejemplo, si las miniciudades están diseñadas para atender las necesidades y anhelos de toda la población. Asimismo, se puede cuestionar el rol de centralidad de estas, a partir del Aleph como lugar donde se encuentran todos los lugares. Los debates que pueden surgir son múltiples, sobre su papel en el comercio y la economía del sistema urbano que se estudie.

\section{Reflexiones finales}

Las diversas aristas de análisis propuestas permiten desvendar que el actual proceso de urbanización requiere de nuevos discursos para conceptualizar, en la teoría y en la práctica, las realidades urbanas y entender las especificidades del urbanismo contemporáneo. De acuerdo con Soja (1996), no es tarea fácil entender la conceptualización del espacio y se acepta la propuesta de salir del histórico rígido dualismo epistemológico que ha caracterizado el análisis espacial.

Las miniciudades, a modo de novedosa tipología, llevan a pensar las dinámicas urbanas más allá del binarismo clásico y, según se vio, permiten formular una diversidad de cuestionamientos sobre diferentes temas. Estas se constataron como un producto inmobiliario y de consumo el cual, a través de la oferta de una simultaneidad de espacios y actividades combinadas, evidencia un cambio del paisaje urbano, del tejido comercial contemporáneo y, por consiguiente, de la combinación de prácticas comerciales en el marco del urbanismo neoliberal (Acosta, 2018).

El análisis de los textos propuestos y las obras de arte seleccionadas demostró lo extraordinario del Third Space o the Other space (el otro espacio) de Soja (1996). La Producción del espacio de Lefebvre (1974), El Aleph de Borges (1949) y El Quijote de Miguel de Cervantes (1966) comprobaron la dificultad que enfrentan el lenguaje, los textos y discursos para capturar la complejidad y diversos significados de las espacialidades; sin embargo, también permiten constatar cómo estos pueden abrir la mente geográfica para el estudio urbano contemporáneo. El objetivo de evidenciar este reto dejó al descubierto que los distintos espacios son escenarios de diversas actividades, donde se combinan diferentes articulaciones de actores y discursos.

Los espacios de uso mixto no son solo para comercio o entretenimiento, sino que abren las posibilidades para combinaciones de actividades. Las miniciudades pueden ser evidencia de nuevos proyectos urbanos que permiten y fomentan cambios en las dinámicas urbanas de ocio, residencia y trabajo. La amplia diversidad de espacios y sus hibridaciones incluye los absolutos, abstractos, concretos, contradictorios, culturales, diferentes, dramatizados, familiares, fragmentados, globales, locales, históricos, homogéneos, heterogéneos, idealizados, imposibles, imaginados, irreales, lujosos, mentales, naturales, neutrales, nuevos, percibidos, perturbados, posibles, reales, representativos, subjetivos, transparentes, utópicos, viejos, verdaderos, y vividos, por mencionar algunos, junto con los propuestos por Soja (2000).

Este artículo permitió iniciar una discusión sobre propuestas de herramientas para analizar y verificar la complejidad de la simultaneidad espacial inmersa en las recientes ciudades reconfiguradas por un urbanismo neoliberal con proyectos como miniciudades. Estas aproximaciones desde la perspectiva de la geografía, la literatura y el arte son solo el punto de partida para enriquecer la imaginación geográfica y abarcar la multiplicidad de visiones sobre los emergentes espacios de los nuevos productos inmobiliarios (Acosta, 2018). Las discusiones permitieron mostrar cómo las recientes tipologías o productos inmobiliarios híbridos obligan a adoptar novedosas perspectivas multidisciplinarias. Son diversas aproximaciones teórico-metodológicas que sirven para también reconsiderar el significado de otros conceptos asociados al fenómeno urbano en futuras discusiones. No se concluye de forma restrictiva ninguna herramienta puntual, sino que se abren muchos más cuestionamientos que ejemplifican de qué manera se puede orientar una investigación geográfica con el apoyo de diferentes disciplinas y fuentes. 


\section{Referencias bibliográficas}

Acosta, S. (2017). "Identificando las miniciudades en América Central: entre barrios cerrados, centros comerciales y parques temáticos", RevistARQUIS, 6 (2), pp.

Acosta, S. (2018). "Miniciudades centroamericanas: rompiendo el dualismo del análisis espacial". Revista IPNUSAC (Instituto de Problemas Nacionales Universidad de San Carlos de Guatemala), (140), pp.

Baudrillard, J. (2016). Cultura y Simulacro. Kairós.

Borges, J. (1949 [2020]). The Aleph. Libros de Mario. https://www.librosdemario.com/ el-aleph-leer-online-gratis/35-paginas

(1999). El hacedor. Alianza Editorial.

Bozzano, H. (2004). Territorios reales, territorios pensados, territorios posibles. Aportes para una teoría territorial del ambiente. Buenos Aires: Espacio Editorial.

Camacho, A. (2013, 4 de agosto). Cuatro miniciudades comerciales emergen en el oeste de la capital. El Financiero. https://www.elfinancierocr.com/negocios/ cuatro-miniciudades-comerciales-emergen-en-el-oeste-de-la-capital/ IR4PIIKFGNGIJKSUSH3M6NKL74/story/.

Cervantes-Saavedra, M. de, 1547-1616. El Ingenioso Hidalgo Don Quijote De La Mancha. Madrid: Espasa-Calpe, 1966.

Derycke, P., Huiriot, J., Pumain, D. (1996). Penser la Ville. Théories et modèles. Anthropos.

Don Drone Guatemala. (2015). Paseo Cayalá, Guatemala. https://www.youtube.com/ watch?v=BuMCG6BwZHo.

Foucault, M. (1984). Des espaces autres. Conferencia dictada en el Cercle des études architecturals, 14 de marzo de 1967, Architecture, Mouvement, Continuité, (5), Traducida por Pablo Blitstein y Tadeo Lima. http://yoochel.org/wp-content/ uploads/2011/03/foucalt_de-los-espacios-otros.pdf

Foucault, M. (2007). Las palabras y las cosas. Siglo XXI.

Gottdiener, M. (1997). The theming of America, Westview Press.

Groover, J. (2015). Dueños y señores del comercio. SCT, Shopping Centers Today Iberoamérica, p.16

Gruen, V. (1960). Shopping towns USA. The planning of shopping centers. Reinhold Publishing Corporation.

Lefebvre, H. (1974). La production de l'espace. Anthropos.

L.M. (2012). Paseo Cayalá Guatemala-Krier, https://mrmannoticias.blogspot.fr/2012/01/ paseo-cayala-guatemala-krier.html

Lizán, J. (2018, junio). Los centros comerciales enfrentando el cambio ¿Qué sigue? Revista Inversión Inmobiliaria. https://www.inversioninmobiliariacr.com/es/ mercado-inmobiliario/comercial/item/1559-los-centros-comerciales-enfrentandoel-cambio-que-sigue.

Lizán, J. (2018b, julio 27). Acerca de Avenida Escazú. En: Avenida Escazú, Todo en un mismo lugar. Inmobiliare. https://inmobiliare.com/avenida-escazu-todo-en-unmismo-lugar/.

Lukas, S. (2007). The Themed space. Locating culture, nation and self. Lexington Books. (2008). Theme park. Reaktion books.

(2013). The immersive worlds handbook. Designing theme parks and consumer places. Focal press.

Monnet, J. Le risque de ghetto dans la ville de demain: le paradoxe du labyrinthe : La recherche de cadres stables et nets dans un monde fluide et flou. EuropéenBelgique. Colloque "La ville de demain », (10), pp.121-131, 2011, Etudes et documents, Aménagement et urbanisme. 
Morales, M. (2016, 13 de diciembre). El este de San José remoza su imagen con vivienda vertical. Inversión Inmobiliaria.http://www.inversioninmobiliariacr.com/ index.php/es/mercado-inmobiliario/residencial/item/727-el-este-de-san-joseremoza-su-imagen-con-vivienda-vertical.

Velazquez, D. (2019). Las Meninas. Museo del Parado. https://www.museodelprado. es/coleccion/obra-de-arte/las-meninas/9fdc7800-9ade-48b0-ab8b-edee94ea877f

National Gallery. (2019). The Arnolfini Portrait, Jan van Eyck. https://www.nationalgallery. org.uk/paintings/jan-van-eyck-the-arnolfini-portrait

Portafolio Inmobiliario. (s.f.). Acerca de Portafolio. http://portafolio.cr/acerca-de-pinmsa/.

Salcedo, R. (2003). Lo local, lo global y el mall: la lógica de la exclusión y la interdependencia. Revista de Geografía Norte Grande. 30(1), 103-115.https:// www.redalyc. org/articulo.oa?id=30003009

Salcedo, R., y De Simone, L. (2013). Una crítica estática para un espacio en constante renovación: El caso del mall en Chile. Revista Atenea, 507, I Sem., 117-132.

Soja, E. (1996). ThirdSpace. Journeys to Los Angeles and other real and imagined places. Blackwell Publishing.

(2000). Postmetropolis, Critical studies of cities and regions. Blackwell Publishing.

Sorkin, M. (1992). See you in Disneyland. En: SORKIN, M. Variations on a Theme Park: The New American City and the End of Public Space. New York: Hill and Wang, pp. 205-232.

Velásquez, D. (1656). Las Meninas. https://www.themasterpiececards.com/famouspaintings-reviewed/famous-painters-diego-velazquez/ 